PERSPECTIVA DE LA EDUCACIÓN AMBIENTAL EN LA GENERACIÓN CENTENNIAL: LA CULTURA DEL RECICLAJE, UN AHORRO ECONÓMICO O CUIDADO DEL MEDIO AMBIENTE.

\title{
PERSPECTIVA DE LA EDUCACIÓN AMBIENTAL EN LA GENERACIÓN CENTENNIAL: LA CULTURA DEL RECICLAJE, UN AHORRO ECONÓMICO O CUIDADO DEL MEDIO AMBIENTE
}

\section{PERSPECTIVE ON ENVIRONMENTAL EDUCATION IN THE CENTENNIAL GENERATION: RECYCLING CULTURE, MONEY SAVING OR ENVIRONMENTAL CARE}

\author{
María Mayela Terán Cázares*, Blanca Nelly Rodríguez Garza**

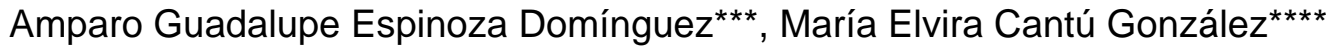

* Doctora. Universidad Autónoma de Nuevo León. Facultad de Contaduría Pública y Administración. Email: mayela.teran@gmail.com. ORCID: http://orcid.org/0000-0001-5089-3909.

** M.E. Universidad Autónoma de Nuevo León. Facultad de Contaduría Pública y Administración. Email: blanca.rodriguezgrz@uanl.edu.mx. ORCID: http://orcid.org/0000-0002-9291-0292.

*** M.A.E. Universidad Autónoma de Nuevo León. Facultad de Contaduría Pública y Administración. Email: guadalupe.espinozad@uanl.mx. ORCID: http://orcid.org/0000-0002-6176-0818.

**** M.A.E. Universidad Autónoma de Nuevo León. Facultad de Contaduría Pública y Administración. Email: maria.cantug@uanl.mx. ORCID: http://orcid.org/0000-0001-6241-4530.

Dirección para recibir correspondencia: mayela.teran@gmail.com 
PERSPECTIVA DE LA EDUCACIÓN AMBIENTAL EN LA GENERACIÓN CENTENNIAL: LA CULTURA DEL RECICLAJE, UN AHORRO ECONÓMICO O CUIDADO DEL MEDIO AMBIENTE.

\section{RESUMEN}

OBJETIVO: Analizar como la educación ambiental desde el contexto teórico, interviene en la conformación de una cultura de reciclaje desde la perspectiva de ahorro o apoyo al medio ambiente en el estudiante Centennial, contribuyendo a su preparación integral con los entornos globales donde se desenvolverá el estudiante al egresar.

MATERIAL Y MÉTODO: Se aplicó un instrumento a un total de 100 estudiantes, que estudiaron el 1er semestre de una universidad privada. Dicha herramienta contiene un total de 45 ítems, en dos secciones, en la primera etapa se encuentra una tabla comparativa con 21 acciones, que indican si pertenecía al ahorro personal o al cuidado de ello, la segunda parte tiene 24 ítems, donde el estudiante responde en una escala de Likert del 1 al 5.

RESULTADOS: Se analizó la primera parte respecto a la perspectiva de ahorro y ayuda al medio ambiente, la cual muestra las atribuciones que el estudiante señala en cada una de las actividades, que predomina su preocupación por el medio ambiente al señalarlo en su puntuación.

Además, se observó la interrelación de la conducta por un cuidado del medio ambiente, a través de componentes de un modelo vinculado a tener un alto compromiso con el apoyo del medio ambiente, a través del ahorro de energía y recursos.

CONCLUSIONES: El desarrollo de la investigación es importante, porque se encontró como en la perspectiva ambiental genera un alto compromiso con el entorno ambiental, formando conductas de apoyo a los recursos naturales.

PALABRAS CLAVE: Educación ambiental. Generación Centennial. Reciclaje.

\section{ABSTRACT}

OBJECTIVE: To analyze how environmental education intervenes in the recycling culture from a theoretical context, and from the money saving or environmental care in centennial students, thus 
PERSPECTIVA DE LA EDUCACIÓN AMBIENTAL EN LA GENERACIÓN CENTENNIAL: LA CULTURA DEL RECICLAJE, UN AHORRO ECONÓMICO O CUIDADO DEL MEDIO AMBIENTE.

contributing to an integral education so graduates can have the expected performance in international scenarios

MATERIAL AND METHOD: An instrument was administered to 100 students from a private university and, who were enrolled in the 1st. semester of their career. The questionnaire had two sections with a total of 45 items, in the first one, there is a comparative table with 21 actions related to personal money saving, the second one had 24 items, and the answers were options in a Likert scale from 1 to 5 .

RESULTS: The first part of the study indicated that when referring to money saving and care for the environment perspective, a concern for the care of the environment is present as well as certain attributions students point out in each of the activities.

Furthermore, a care behavior for the environment was observed through elements of a model linked to a high commitment for the support of the environment, and also related to energy and resources conservation.

CONCLUSIONS: The development of the research is relevant because the environmental perspective generates a high commitment to the environment, thus creating supporting behavior to natural resources.

KEY WORDS: Environmental educaton. Centennial generation. Recycling.

\section{INTRODUCCIÓN}

Las universidades, como seres formadores de sociedades integrales y comprometidas como un todo en un mundo globalizado, deben reflexionar sobre su papel que conforma su influencia en la formación de los actores que intervienen en el proceso de educación ambiental generadora de conductas, hábitos y costumbres, partícipes de un entorno sociocultural y que influye en las decisiones organizacionales.

TERÁN-CÁZARES M. M., RODRÍGUEZ-GARZA B. N., ESPINOZA-DOMÍNGUEZ A. G., CANTÚ-GONZÁLEZ M. E. 
PERSPECTIVA DE LA EDUCACIÓN AMBIENTAL EN LA GENERACIÓN CENTENNIAL: LA CULTURA DEL RECICLAJE, UN AHORRO ECONÓMICO O CUIDADO DEL MEDIO AMBIENTE.

A partir de la Conferencia de las Naciones Unidas en 1972, se da inicio por crear conciencia sobre la relación que existe entre las dimensiones, económicas, sociales y ambientales, las cuales crean un vínculo de supervivencia para la sociedad (Bebbington, Russell \& Thompson, 2017).

Las conductas sostenibles son enmarcadas por Tapia et.al (2013), donde destacan actividades destinadas al cuidado de los recursos naturales, asimismo las conductas frugales, demandan todo aquel acto que disminuya el consumo y desperdicio de recursos, de esta manera los comportamientos equitativos señalan una distribución de materiales los cuales sean justos.

De esta manera, D’Amato (2004), señala básicamente que el comportamiento, las actitudes y motivaciones del hombre se ven intrínsecamente ligados a su relación con los otros y con su medio...las diversas formas de interrelación con la naturaleza, los hábitos de sustancia y los estilos de vida se convierten en fuentes de información para crear programas de educación ambiental.

En ese sentido, la cultura ambiental es definida por Mendoza et al. (2019), como "la forma en como la sociedad adopta medidas para promover una relación estable con el ambiente, y se orienta principalmente a procesos sociales, políticos, económicos y culturales" (p. 39).

Hoy en día, en el ámbito educativo, la generación Centennial, ha sido motivo de numerosos estudios, actualmente se encuentran en el nivel básico, intermedio y superior de la educación; de manera general, se pueden observar características, tales como: los expertos que son en el uso de herramientas tecnológicas, pueden realizar varias actividades a la vez, son multitareas, se relacionan socialmente a través de las tecnologías, son impacientes, interactivos y resilientes, a la vez preocupados por su salud y al medio ambiente.

\section{Pregunta central de investigación}

¿Cómo intervienen en el proceso de educación ambiental el contexto de cultura del reciclaje, desde la perspectiva del cuidado del medio ambiente o ahorro económico?

\section{Objetivo general}

Analizar como la educación ambiental desde el contexto teórico, interviene en la conformación de una cultura de reciclaje, desde la perspectiva de ahorro o apoyo al medio ambiente en el 
PERSPECTIVA DE LA EDUCACIÓN AMBIENTAL EN LA GENERACIÓN CENTENNIAL: LA CULTURA DEL RECICLAJE, UN AHORRO ECONÓMICO O CUIDADO DEL MEDIO AMBIENTE.

estudiante Centennial, contribuyendo a su formación integral acorde a los entornos globales donde se desenvolverá el estudiante al egresar.

\section{MARCO TEORICO}

\section{Educación Ambiental}

La educación ambiental es definida por Sauvé (2014), como la capacidad de ser responsable a nivel colectivo en cuanto a las competencias que requieren una involucración eficiente en los espacios ambientales, con la finalidad de ejercer acciones consientes sobre los asuntos socioecológicos.

Los objetivos de la educación ambiental son marcados por González (2001), destacando principalmente:

- Lograr que los actores sociales, en forma individual o colectiva, comprendan la naturaleza compleja del medio ambiente natural, así como del creado por el hombre, que es el resultado de la interacción de sus aspectos biológicos, físicos, sociales, económicos y culturales.

- Adquirir los valores, la conducta, cambio de cultura, comportamiento y habilidades prácticas para prevenir y solucionar los problemas ambientales.

Desde la perspectiva educativa, el medio ambiente como campo de formación del individuo es trascendental ya que, a través de un programa de estudio, planes analíticos mediante resultados o competencias se pueden formar personas más conscientes de la problemática medio ambiental actual (Marcillo, et al., 2016).

Es por lo que poder formar un aprendizaje sobre el cuidado del medio ambiente debe de considerar actitudes, conocimiento y conductas encaminadas al reciclaje, asimismo se debe a aprender a generar actividades que den paso a la importancia de la recolección de reciclables que faciliten el entorno (Vicente \& Reis, 2007).

Sin embargo, Leff (2009), señala la importancia de formar una cultura ecológica que se caracteriza por "la mediación entre la sociedad y el medio ambiente, combinando los diversos estilos étnicos propios de cada individuo y el aprovechamiento racional de los recursos naturales" (p. 221). 
PERSPECTIVA DE LA EDUCACIÓN AMBIENTAL EN LA GENERACIÓN CENTENNIAL: LA CULTURA DEL RECICLAJE, UN AHORRO ECONÓMICO O CUIDADO DEL MEDIO AMBIENTE.

El reciclar se establece como "cualquier proceso donde residuos o materiales de desperdicios son recolectados y trasformados en nuevos materiales que pueden ser utilizados o vendidos como nuevos productos o componentes primos" (Bonilla, 2016, p. 42).

Asimismo, Bayón y Morejón (2007), indican que la política y la educación juegan un papel importante ya que pueden formar regulaciones en cuanto a la relación de las acciones humanas con la naturaleza, esto por medio de la cultura a partir del entorno ambiental, creando una dinámica que fortalezca la sustentabilidad.

Es importante señalar que existen diferentes teorías psicológicas, las cuales generan comportamientos y conductas para generar una disminución en el deterioro ambiental, las cuales destacan:

\section{El Conductismo}

Destaca por ser una teoría la cual tiene como principal objetivo, el estudiar las acciones que son derivadas de la conducta y se pueden detectar fácilmente (Skiner, 1953). A partir de esta descripción, se vincula el aspecto ambiental debido a el modelo de la triple relación de contingencias, en donde Corral, et al. (2013), indican que "un comportamiento es contingente a la aparición de un estímulo discriminativo un evento ambiental que indica la ocasión para que se produzca un comportamiento" (p. 2). El conductismo determina que es más probable que la sociedad actúe bajo los principios anti-ambientalistas que pro ecológicamente (Cone \& Hayes, 1980).

\section{Psicología Evolucionista}

Señala que la conducta, así como los procesos psicológicos son derivados de adaptaciones, resultados de la selección natural en donde estas se evolucionan con la finalidad de dar una solución a los problemas ambientales que surgen (Barkow, Cosmides \& Tooby, 1992). Con respecto a ello Fiala (2008), indica que, debido a la falta de percepciones e inclinaciones hacia el cuidado del medio ambiente, es que día a día aumentan los problemas ecológicos, una solución ante ello la formulan (Sexton \& Sexton, 2014), donde se deban formar actividades proambientales para que formen parte de las normas y así con inversiones económicas se conviertan en conductas pro-sustentables, para que puedan funcionar como estatus y se multipliquen en conductas sostenibles. 
PERSPECTIVA DE LA EDUCACIÓN AMBIENTAL EN LA GENERACIÓN CENTENNIAL: LA CULTURA DEL RECICLAJE, UN AHORRO ECONÓMICO O CUIDADO DEL MEDIO AMBIENTE.

\section{Teorías Cognitivas}

Se indica a partir de Corral en conjunto con otros autores (2019). "básicamente que la orientación de valores que tenga la persona ejercerá una influencia directa sobre las creencias, y, por tanto, sobre la actitud y la conducta... por lo tanto, la persona desarrollará unas creencias más positivas hacia dicha situación, objeto u acción" (p. 3). A partir de este modelo se pueden establecer relaciones de casualidad entre las variables que pueden determinar las diferentes conductas ecológicas que generen responsabilidad (Aguilar, et al., 2012). Asimismo, Corraliza \& De Berenguer (2000), identifican que la conducta ambientalista se crea a partir de los valores (derivados de la activación de normas y obligaciones morales) y las creencias ambientales (en función de beneficios y conductas).

\section{Teoría de Foco Normativo}

Se genera a partir de las influencias de un grupo social sobre el comportamiento ambiental (Cialdini, Reno \& Kallgren, 1990). El cual se construye a partir de definiciones encaminadas a las normas para generar una explicación sobre los comportamientos sociales (Corral, et al., 2019). De esta manera, esta teoría explica los comportamientos anti-ambientales en donde los más significativos son: las normas descriptivas y las prescriptivas que contribuyen a conceptualizar por qué la sociedad no lleva a cabo acciones que van en contra del ambiente (Martínez, et al., 2014).

\section{Teorías de la Acción Razonada (TAR) y Planificada (TCP)}

Uno de sus principales precursores Ajzen (1985). Establece que los controles conductuales son percibidos, así como las intenciones permiten interactuarse en cuanto a la predicción de la conducta, en donde gracias a la eficacia predicen diferentes comportamientos en especial a conductas ecológicas responsables (Aguilar, et al., 2012).

\section{Teorías motivacionales: Autoeficacia y Autorregulación}

La autoeficacia hace referencia a la confianza que posee la persona en su capacidad para afrontar una determinada situación (Bandura, 1977), reflejando el control que el individuo cree tener sobre las circunstancias que afectan a su vida. Recientemente se ha incorporado la autoeficacia en la explicación de la conducta pro-ambiental; las conductas autorreguladas y autoTERÁN-CÁZARES M. M., RODRÍGUEZ-GARZA B. N., ESPINOZA-DOMÍNGUEZ A. G., CANTÚ-GONZÁLEZ M. E. 
PERSPECTIVA DE LA EDUCACIÓN AMBIENTAL EN LA GENERACIÓN CENTENNIAL: LA CULTURA DEL RECICLAJE, UN AHORRO ECONÓMICO O CUIDADO DEL MEDIO AMBIENTE.

determinadas, proveen al individuo de motivación automática que garantizaría el mantenimiento de sus comportamientos sustentables (Corral, et al., 2019).

\section{Generación Centennial}

Nacidos a partir del año 1995, según Wood (citado por Dutra, 2017), los miembros de la generación Centennial están caracterizados por vivir en un mundo globalizado, haciendo parecer el mundo como un lugar más pequeño y accesible, con acelerados cambios sociales y tecnológicos que impactan en la subjetividad de los jóvenes. Esta Generación, es la única que puede considerarse como plenamente global, esta variante hace que las diferencias propias de las sociedades que habitan el mundo no sean tan diversas como en el resto de las generaciones (Dutra, 2017).

El problema radica en la capacidad del docente para imaginar la vida laboral de acuerdo a un nuevo entorno ambiental y cultural del cual personal y socialmente es parte del estudiante e incorporar esta visión al trabajo del aula, el profesor, debe mantenerse actualizado en el uso de las nuevas tendencias globales en materia de medio ambiente del que sus alumnos con los avances tecnológicos ya se encuentran informados.

De acuerdo con Maioli, y Filipuzzi, (2016), algunas de las características de esta Generación son:

- Desarrollan habilidades autodidactas, donde YouTube es uno de sus principales recursos a través de tutoriales.

- Son de la idea de vivir una experiencia profesional que les satisfaga, afín a su forma de ver y comprender el mundo que habitan.

- Su aspiración profesional es adquirir conocimientos y experiencias que les permitan enfrentar nuevos desafíos profesionales.

- Buscan nuevos desafíos, buen ambiente laboral, y la posibilidad de desarrollar su carrera profesional.

- Viven el cambio como algo inherente a su vida.

- Sus aspiraciones plantean desafíos de la necesidad de un alineamiento con los valores corporativos.

- Utilizan internet tanto para buscar empleo; como en su formación personal y profesional, así como para interactuar con marcas. 
PERSPECTIVA DE LA EDUCACIÓN AMBIENTAL EN LA GENERACIÓN CENTENNIAL: LA CULTURA DEL RECICLAJE, UN AHORRO ECONÓMICO O CUIDADO DEL MEDIO AMBIENTE.

- Suelen ser fieles a las marcas, aunque ponen más atención en la inmediatez de su necesidad que en la calidad y asumen un uso efímero de lo comprado.

Silvestre y Cruz (2016) afirman que la Generación Centennial o Generación Z, ha iniciado sus estudios de nivel superior y está influyendo en los métodos de enseñanza de las universidades. Estos jóvenes tienden a entornos de aprendizaje colectivo, donde pueden estar directamente involucrados en el proceso de aprendizaje.

El poder tener acceso a una nueva información ilimitada ha creado una generación más autosuficiente y autodidacta. De hecho, el 13\% de los integrantes de la Generación Z, ya tienen su propio negocio y muchos están, incluso, tomando este espíritu emprendedor para impulsar los cambios en el plan de estudios de la universidad, toda vez que muestran un fuerte interés en el diseño de su propio camino de clase en la universidad (Olivares y González, 2016).

\section{MATERIAL Y MÉTODO}

La presente investigación es descriptiva, correlacional y explicativa, de forma transversal considerando el estudio de manera cuantitativa y no experimental. La muestra se integró por 100 estudiantes universitarios que cursaron el 1er semestre en una institución privada de educación superior de un universo de 160 alumnos inscritos en ese período. El instrumento utilizado fue conformado con 45 ítems en 2 secciones; en la primera, se utilizó una tabla comparativa con 21 aseveraciones o actividades, de las cuáles el participante señalaba en una sola opción si esta la identificaba como ahorro personal o apoyo al medio ambiente, mientras que la segunda sección, está compuesta por 24 ítems y se utilizó la escala de Likert de 1 al 5 para su respuesta, en donde se pretende conocer que tan de acuerdo está, donde 1 es totalmente en desacuerdo, 2 en desacuerdo, 3 ni de acuerdo ni en desacuerdo, 4 de acuerdo y 5 totalmente de acuerdo. En los datos demográficos recopilados se analizó género, edad, municipio, número de hermanos, posición en la familia que ocupa, intercambios en el extranjero, participación en labores sociales o ambientalistas y grupos a los que pertenece (deportivo, religioso).

Dentro del procedimiento que se siguió, se permitió generar y validar un instrumento para medir las variables del estudio propuestas; en la primera etapa se desarrolló una revisión bibliográfica para diseñar los ítems y adaptar los mismos. En la segunda etapa, se procedió a realizar una validez de apariencia, en donde se revisó y reestructuró la redacción de cada uno de los ítems 
PERSPECTIVA DE LA EDUCACIÓN AMBIENTAL EN LA GENERACIÓN CENTENNIAL: LA CULTURA DEL RECICLAJE, UN AHORRO ECONÓMICO O CUIDADO DEL MEDIO AMBIENTE.

por parte de población vinculada con el estudio. En la tercera etapa, se aplicó a la muestra identificada, lo cual permitió validar a través del Alpha de Cronbach los resultados obtenidos. Dando pie a generar un instrumento para futuras investigaciones y determinar la perspectiva de la cultura de reciclaje para diferentes tipos de población.

\section{RESULTADOS}

De acuerdo a los resultados descriptivos obtenidos en la presente investigación, se analizó la primer parte respecto a la perspectiva de ahorro o ayuda al medio ambiente, encontrando en la figura 1, los resultados generados en la presente investigación, la cual muestra las atribuciones que el estudiante señala en cada una de las actividades, que predomina su preocupación por el medio ambiente al señalarlo en su puntuación.

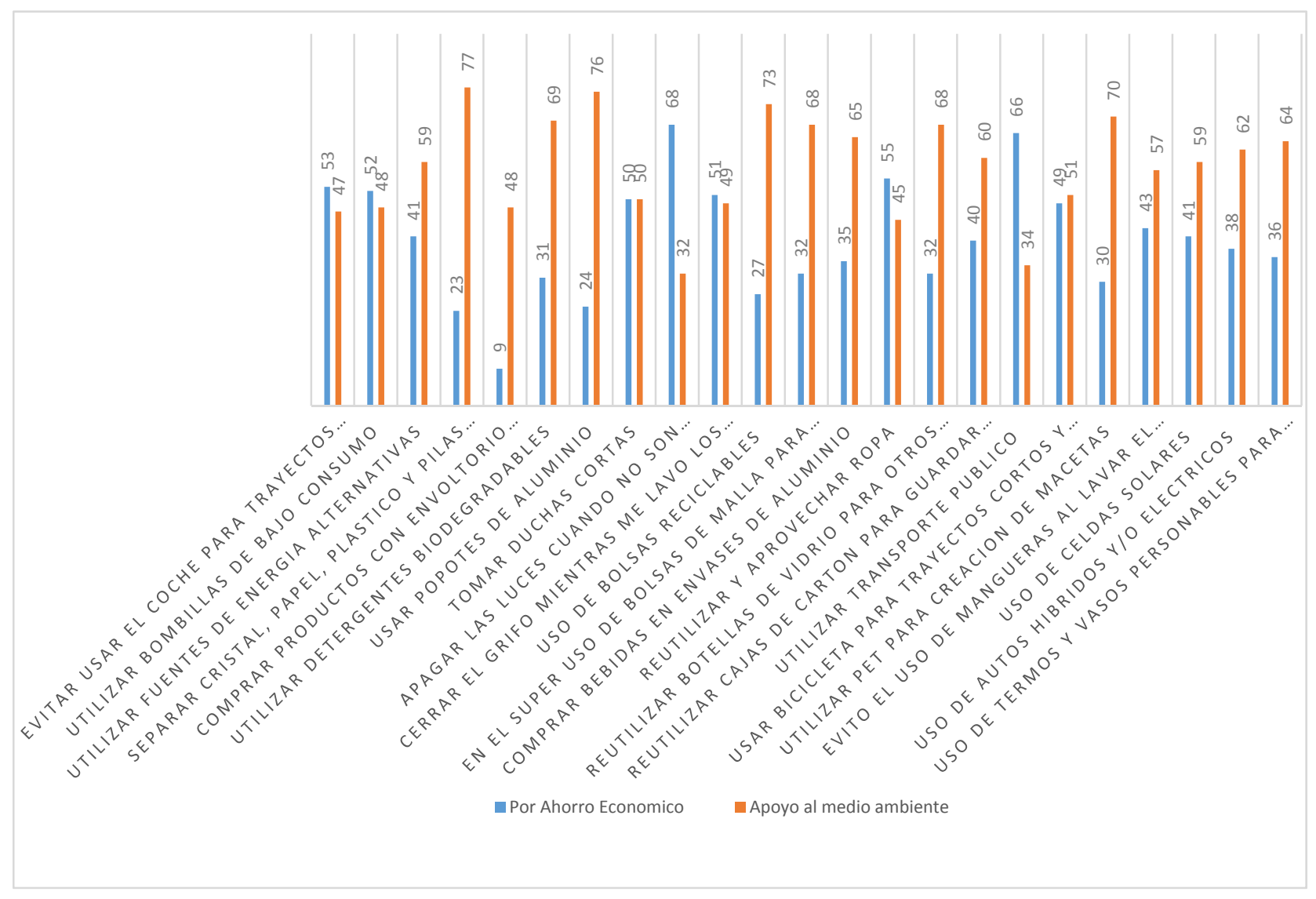

Figura 1. Análisis de la perspectiva del ahorro y apoyo al medio ambiente.

Fuente: Elaboración propia.

TERÁN-CÁZARES M. M., RODRÍGUEZ-GARZA B. N., ESPINOZA-DOMÍNGUEZ A. G., CANTÚ-GONZÁLEZ M. E. 
PERSPECTIVA DE LA EDUCACIÓN AMBIENTAL EN LA GENERACIÓN CENTENNIAL: LA CULTURA DEL RECICLAJE, UN AHORRO ECONÓMICO O CUIDADO DEL MEDIO AMBIENTE.

En la segunda fase, se analizó mediante el Alpha de Cronbach la fiabilidad del instrumento, observándose en la tabla 1, los resultados arriba de .7, lo que demuestra que los datos recopilados tienen la validez de acuerdo a los ítems colocados en cada uno de los constructos.

Tabla 1

Alpha de Cronbach's fase 2

\begin{tabular}{cc}
\hline Cronbach's Alpha & N of Items \\
\hline .834 & 15 \\
.837 & 7 \\
.800 & 12 \\
\hline
\end{tabular}

Fuente: Elaboración propia.

En esta segunda fase, se observó la interrelación de la conducta por un cuidado del medio ambiente, a través de componentes de un modelo vinculado a tener un alto compromiso con el apoyo del medio ambiente, a través del ahorro de energía y recursos.

\section{CONCLUSIONES}

De acuerdo al análisis desarrollado, se observó que en la mayoría de las actividades presentadas, se realizan beneficios del medio ambiente más que un ahorro económico, lo que puede ser atribuido al tipo de generación que fue encuestado, ya que una de sus características que los distinguen es su preocupación y cuidado del medio ambiente, con esto se puede percibir que esta generación piensa en el planeta, y que al momento de realizar alguna actividad, en lugar de pensar en una conveniencia propia, buscan la manera de tener un resultado equilibrado para generar un ahorro económico, tales como: compartir el automóvil para trayectos largos o reducir el tiempo en la ducha, los cuales son atribuibles a culturas o frases, que a través del tiempo se han enmarcado como una necesidad económica y ambiental.

Las actividades que predominaron por un número mayor en la opción de ahorro económico fueron, apagar las luces cuando no son necesarias y utilizar el transporte público, esto se puede asociar un poco más a los precios elevados del combustible y la electricidad y no reforzándose en cuidados ambientales.

En base al análisis anterior, es importante la reflexión de entender si se está educando a un profesionista sensible a las necesidades de un medio ambiente, con una capacidad integral de liderar y ser partícipes de un cambio cultural, en donde el cambio sea la base del cumplimiento TERÁN-CÁZARES M. M., RODRÍGUEZ-GARZA B. N., ESPINOZA-DOMÍNGUEZ A. G., CANTÚ-GONZÁLEZ M. E. 
PERSPECTIVA DE LA EDUCACIÓN AMBIENTAL EN LA GENERACIÓN CENTENNIAL: LA CULTURA DEL RECICLAJE, UN AHORRO ECONÓMICO O CUIDADO DEL MEDIO AMBIENTE.

de objetivos y metas en la dinámica de las nuevas organizaciones. Además, crear en ello conductas innatas que puedan ser replicadas de manera natural en un ambiente global, esto a través de la educación ambiental, lo cual es un gran reto y los resultados se dieron al inicio de esta investigación.

\section{REFERENCIAS BIBLIOGRÁFICAS}

Aguilar, M., García, J., Calvo, A. \& Salinas, J. (2012). Comparative study between the theory of planned behaviour and the value-belief-norm model regarding the environment on Spanish housewives' recycling behavior. Journal of Applied Social Psychology, 42, 27972833. DOI: 10.1111/j.1559-1816.2012.00962.x

Ajzen, I. (1985). From intention to action: A theory of planned behavior. Recuperado de https://www.scirp.org/(S(i43dyn45teexjx455qlt3d2q))/reference/ReferencesPapers.aspx ?ReferenceID=940613

Astudillo, E., Melendres, N., Espinoza, B., Moscoso, M. y Baquerizo, T. (2015). Cultura ecológica de jóvenes universitarios. Investigatio, 6, 123-140. Recuperado de http://revistas.uees.edu.ec/index.php/IRR/article/view/27/26

Bandura, A. (1997). Self-efficacy: The exercise of control. Recuperado de https://psycnet.apa.org/record/1997-08589-000

Barkow, J., Cosmides, L. \& Tooby, J. (1992). The adapted mind: Evolutionary psychology and the generation of culture. Recuperado de http://lolita.unice.fr/ scheer/cogsci/Barkow\%20et\%20al.\%20(eds.)\%2092\%20(EXTRAIT S)\%20\%20The\%20Adapted\%20Mind.\%20Evolutionary\%20Psychology\%20and\%20the \%20Generation\%20of\%20Culture.pdf

Bayón, P. y Morejón, A. (2007). Cultura Ambiental y la Construcción de Entornos de Reproducción Social en Cuba. Grupo GEMAS, 2(3), 1-9. Recuperado de http://biblioteca.clacso.edu.ar/ar/libros/cuba/if/marx/documentos/22/Cultura\%20ambient al\%20y\%20la\%20construcci\%F3n\%20de\%20entornos\%20de....pdf

Bebbington, J., Russell, S. \& Thomson, I. (2017). Accounting and sustainable development: Reflections and propositions. Critical Perspectives on Accounting, 42, 21-34. Recuperado de https://ideas.repec.org/a/eee/crpeac/v48y2017icp21-34.html

Bonilla, D. (2016). El reciclaje como estrategia didáctica para la conservación ambiental. Revista Scientific, 1(1), 35-52. DOI: 10.29394/scientific.issn.2542-2987.2016.1.1.3.36-52 
PERSPECTIVA DE LA EDUCACIÓN AMBIENTAL EN LA GENERACIÓN CENTENNIAL: LA CULTURA DEL RECICLAJE, UN AHORRO ECONÓMICO O CUIDADO DEL MEDIO AMBIENTE.

Cerezo, P. (2017). La Generación Z y la información: Ios auténticos nativos digitales: ¿estamos preparados para la Generación Z? Revista de Estudios de Juventud, 16(114), 95-109. Recuperado de http://www.injuve.es/sites/default/files/2017/28/publicaciones/documentos_7._la_gener acion_z_y_la_informacion.pdf

Cialdini, R., Reno, R. \& Kallgren, C. (1990). A focus theory of normative conduct: recycling the concept of norms to reduce littering in public places. Journal of Personality and Social Psychology, 58, 1015-1026. DOI: 10.1037/0022-3514.58.6.1015

Cone, J. \& Hayes, S. (1980). Environmental problems; Behavioral solutions. Recuperado de https://trove.nla.gov.au/work/9686973?q\&versionld=21464637

Corral, V., Aguilar, M. y Hernández, B. (2019). Bases teóricas que guían a la psicología de la conservación ambiental. Papeles del Psicólogo, 40(3), 174-181. DOI: 10.23923/pap.psicol2019.2897

Corraliza, J. \& De Berenguer, J. (2000). Environmental values, beliefs and actions: A situational approach. Environment and Behavior, 32(6), 832-848. DOI: $10.1177 / 00139160021972829$

D'Amato, G. (2004). Psicología Socioambiental, representaciones sociales y educación ambiental. Informes Psicológicos, 6, 67-72. Recuperado de https://revistas.upb.edu.co/index.php/informespsicologicos/article/view/6104/5599

Dutra, M. (2017). Generación Z: entre las nuevas formas de organización del trabajo y la convivencia generacional. Recuperado de https://www.colibri.udelar.edu.uy/jspui/bitstream/123456789/10918/1/Dutra\%2C\%20Ma. \%20Florencia.pdf

Fiala, N. (2008). Meeting the demand: An estimation of potential future greenhouse gas emissions from meat production. Ecological Economics, 67(15), 412-419. DOI: 10.1016/j.ecolecon.2007.12.021

González, E. (2001). Otra lectura a la historia de la educación ambiental en América Latina y el Caribe. Desenvolvimento e Meio Ambiente, 3, 141-158. Recuperado de http://www.ecologiasocial.com/biblioteca/GonzalezGhisotiraEducAmbALat.pdf

Leff, E. (2004). Racionalidad Ambiental: La reapropiación social de la naturaleza. Recuperado de http://ru.iis.sociales.unam.mx/jspui/bitstream/IIS/4937/1/Racionalidad_ambiental.pdf 
PERSPECTIVA DE LA EDUCACIÓN AMBIENTAL EN LA GENERACIÓN CENTENNIAL: LA CULTURA DEL RECICLAJE, UN AHORRO ECONÓMICO O CUIDADO DEL MEDIO AMBIENTE.

Marcillo, F., Marcillo, D., Soledispa, X. y Caicedo, C. (2016). Contribución de las TIC en la problemática medio ambiental y su incidencia en instituciones de educación superior. $3 C$ Empresa, 5(4), 41-57. Recuperado de https://dialnet.unirioja.es/servlet/articulo?codigo $=5746480$

Martín, A., Hernández, B., Frías M., \& Hess, S. (2014). Why ordinary people comply with environmental laws: A structural model on normative and attitudinal determinants of illegal anti ecological behaviour. Legal and Criminological Psychology, 19, 80-103. DOI: 10.1111/j.2044-8333.2012.02062.x

Mendoza, H., Loayza, M. y González, M. (2019). Análisis de la Gestión Ambiental como indicador de la responsabilidad social universitaria. Universidad y Sociedad, 11(2), 3741. Recuperado de https://rus.ucf.edu.cu/index.php/rus/article/view/1147/1201

Olivares, S. y González J. (2016). La generación Z y los retos del docente. En I. J. Velasco y M. Páez (coord.), Los retos de la docencia ante las nuevas características de los estudiantes universitarios (pp. 114-124). Nayarit, México: ECORFAN.

Sauvé, L. (2014). Educación ambiental y ecociudadania. Dimensiones claves de un proyecto político-pedagógico. Revista Científica, 1(18), 13-23. DOI: 10.14483/23448350.5558

Sexton, S. y Sexton, A. (2011). Conspicuous conservation: the Prius effect and willingness to pay for environmental bona fides. Journal of Environmental Economics and Management, 67(3), 303-317. DOI: 10.1016/j.jeem.2013.11.004

Silvestre, E. y Cruz, O. (2016). Conociendo la próxima generación de estudiantes universitarios dominicanos a través de las redes sociales. Ciencia y Sociedad, 41(3), 475-503.

Skinner, B. (1953). Science and human behavior. Recuperado de https://onlinelibrary.wiley.com/doi/abs/10.1002/sce.37303805120

Tapia, C., Corral, V., Fraijo, B. y Durón, F. (2013). Assessing sustainable behavior and its correlates: a measure of pro-ecological, frugal, altruistic and equitable actions. Sustainability, 5(2), 711-723. DOI: 10.3390/su5020711

Vicente, P. \& Reis, E. (2007). Segmenting households according to recycling attitudes in a Portuguese urban area. Resources Conservation and Recycling, 52(1), 1-12. DOI: 10.1016/j.resconrec.2007.01.005 